\title{
Studies on Long-Term Depression in Area CA1 of the Anesthetized and Freely Moving Rat
}

\author{
Ursula Staubli and Joey Scafidi \\ Center for Neural Science, New York University, New York, New York 10003
}

\begin{abstract}
Homosynaptic long-term depression (LTD) is reported to occur in field CA1 of hippocampal slices collected from immature brains. Because the effect has been postulated to be a memory storage mechanism, it is of interest to test for its presence in adult, awake animals. Unfortunately, not only has hippocampal LTD proved difficult to obtain reliably in vivo, but the few successful studies vary with respect to protocols and evidence that the depression is input-specific. The present study tested for input-specific (homosynaptic) LTD in field CA1 after application of various stimulation protocols to the Schaffer collateral/ commissural projections in freely moving, adult rats. The results indicate that although low-frequency trains do induce decrements in synaptic transmission lasting for hours to several days, the success rate of eliciting input-specific LTD in the awake rat is very modest compared with the ease with which
\end{abstract}

stable potentiation is obtained in the same synapses. Moreover, it is questionable that the effective protocols represent patterns of activity likely to occur during behavior. The stronger the afferent activation during low-frequency stimulation, the greater was the probability of eliciting LTD accompanied by persistent heterosynaptic depression. Clear evidence for the occurrence of LTD, irrespective of stimulation protocol and current intensity, could not be obtained in rats under barbiturate anesthesia. In all, the results do not accord with the suggestion that LTD occurs routinely in the hippocampus in vivo as part of memory encoding.

Key words: hippocampus; CA1; long-term depression; homosynaptic; heterosynaptic; low-frequency stimulation; long-term potentiation
A depressive counterpart to long-term potentiation (LTP) has considerable theoretical significance with respect to issues regarding memory saturation and possible causes for forgetting. Activitydependent synaptic depression also occupies a central role in many neuronal network models that incorporate the weakening of synapses into their learning algorithms (Bienenstock et al., 1982; Hopfield, 1982). One example of an activity-dependent decrease in synaptic efficacy is the phenomenon of homosynaptic long-term depression (LTD), which has become a topic of great interest in recent years. LTD is defined as a persistent decrease in slope and amplitude of the evoked response in naive (unpotentiated) pathways typically obtained by applying minutes-long trains of singlepulse stimulation at a rate of $1-3 \mathrm{~Hz}$ (for review, see Bear and Malenka, 1994). The effect is more reliably induced and robust in hippocampal slices prepared from immature rats than in slices collected from adult animals (Dudek and Bear, 1992, 1993; Staubli and Ji, 1996).

Most of our understanding in LTD of hippocampus comes from in vitro work conducted in area CA1, and the extent to which this information is applicable to the intact brain remains uncertain. Indeed, the occurrence of LTD in vivo has been questioned. There are a number of reports of failed attempts to elicit homosynaptic LTD in the intact animal in response to prolonged single-pulse stimulation at low frequency (Barrionuevo et al., 1980; Staubli and Lynch, 1990; Errington et al., 1995; Staubli et al., 1995; Doyere et al., 1996; Doyle et al., 1997). One possible explanation for the

Received Dec. 23, 1996; revised March 24, 1997; accepted March 27, 1997

This research was supported in part by a New York University Whitehead fellowship to U.S.

Correspondence should be addressed to Dr. Ursula Staubli, New York University, Center for Neural Science, 4 Washington Place, New York, NY 10003

Copyright (C) 1997 Society for Neuroscience $0270-6474 / 97 / 174820-09 \$ 05.00 / 0$ discrepancy between the in vitro versus in vivo results is that the optimal protocol for producing LTD in the intact rat differs from that used in slices. That this might be the case has been suggested by Thiels and colleagues, who used a novel stimulation protocol consisting of patterned pulses to elicit LTD in area CA1 of the anesthetized, adult rat (Thiels et al., 1994). In a follow-up experiment that involved slightly modified stimulation parameters, they were also able to produce LTD in the perforant path-dentate pathway of the unanesthetized rabbit (Thiels et al., 1996). A similar study conducted by Doyere and colleagues (Doyere et al., 1996) obtained reliable and persistent LTD in area CA1 of the awake rat with a modified version of the Thiels protocol.

Although the results summarized above indicate that some form of LTP occurs in vivo, questions remain about (1) the likely relevance of the effect to normal brain operations, and (2) the relationship between depression and LTP reversal (depotentiation). Insight into the first issue will require data on the number and strength of the stimulation pulses needed to elicit robust LTD, the duration of depression, and the extent to which lasting variants can be produced in the absence of generalized changes. The present studies were performed to address these points. A second set of experiments tested for similarities between the substrates of LTD and depotentiation. Relationships between the two phenomena, although of evident importance for ideas of how each might be involved in behavior, have yet to be resolved. Accordingly, tests were performed to determine whether potent drugs that do (up modulators of AMPA receptors) and do not (barbiturates) affect depotentiation have comparable effects on LTD.

\section{MATERIALS AND METHODS}

Subjects. Male Long-Evans rats, 3 months of age at the time of surgery, were used. The animals were housed individually and kept in a 12:12 
light/dark cycle with food and water available ad libitum. The animal care and use protocol for the present study was approved by the New York University Animal Welfare Committee.

Preparation for chronic hippocampal physiology. Preparation of animals with chronically implanted electrodes followed procedures essentially as described in previous work (Staubli and Lynch, 1987, 1990). Subjects were anesthetized deeply with a mixture of ketamine and xylazine (or pentobarbital, in later experiments) and pretreated with atropine to prevent excessive salivation, after which a Teflon-insulated platinum/iridium recording electrode $(75 \mu \mathrm{m})$ was lowered under stereotaxic guidance into stratum radiatum of field CA1 (coordinates, $3.8 \mathrm{~mm}$ posterior and $2.9 \mathrm{~mm}$ lateral to bregma). Two Formvar-coated stainless-steel monopolar stimulating electrodes $(125 \mu \mathrm{m})$ were positioned into field CA3 $(3.5 \mathrm{~mm}$ posterior and $3.5 \mathrm{~mm}$ lateral to bregma), ipsilateral and contralateral to the recording electrode to activate the Schaffer collateral and commissural projections. Most of the rats used for testing interactions between barbiturates and LTD induction (11 of 15) were implanted using slightly modified coordinates according to Heynen et al. (1996) as follows: recording electrode, $3.5 \mathrm{~mm}$ posterior and $2.5 \mathrm{~mm}$ lateral to bregma; stimulating electrode, $3.5 \mathrm{~mm}$ posterior and $2.8 \mathrm{~mm}$ lateral to bregma. An indifferent electrode (125 $\mu \mathrm{m}$, Formvar-coated stainless-steel) with $2 \mathrm{~mm}$ insulation removed from the tip was lowered anterior to the hippocampus, and a skull screw over the cerebellum served as a ground. Physiological recordings were used to adjust the position of the electrodes to maximize the amplitude of the negative-going dendritic field EPSPs elicited by single stimulation pulses to the ipsilateral and contralateral stimulating electrodes. After these steps, the leads of the electrodes were connected to a headstage that was affixed permanently to the rats' skull.

LTD induction in the freely moving rat. Ten days after surgery, the animals were acclimated to a chronic recording cage $(30 \times 30 \times 58 \mathrm{~cm})$ and to the attachment of a recording lead to the headstage. The biphasic stimulation pulses were provided by custom-built computer-operated digital stimulator that allows precise control of current intensity and duration. Recording sessions began by adjusting current intensity (25-100 $\mu \mathrm{A})$ and pulse width $(150-250 \mu \mathrm{sec})$ to produce a response that was $50-60 \%$ of the maximum amplitude of the population spike-free response that typically ranged between 4 and $6 \mathrm{mV}$. Recording signals were preamplified $10 \times$ via a FET operational amplifier built into the recording lead and fed into a second stage amplifier set to a gain of 10 , with a bandpass of $1 \mathrm{~Hz}$ to $5 \mathrm{kHz}$. The evoked responses were monitored on a storage oscilloscope and fed into a PC computer (Dell 386, running customized software with Keithley Metrabyte interface board) that digitized a $30 \mathrm{msec}$ sweep at $10 \mathrm{kHz}$, measured initial slope and peak amplitude of each response, and stored the average waveform of groups of four successive responses on disk for off-line analysis. Baseline evoked EPSPs were tested alternately on the two electrodes at 15-20 sec intervals during baseline periods and after attempts to induce LTD. Baseline periods were conducted daily for 30-45 min for at least $3 \mathrm{~d}$, and only animals with $\leq 10 \%$ variability in size of evoked responses (initial slope and amplitude) over three consecutive days were used. Approximately $75 \%$ of the animals implanted met this criterium. Various low-frequency stimulation (LFS) protocols were tested for their ability to elicit LTD in the awake rat: (1) 900 stimuli at $1 \mathrm{~Hz}$; (2) 3000 stimuli at $5 \mathrm{~Hz}$; (3) 3000 stimuli at $10 \mathrm{~Hz}$; (4) three trains of 3000 stimuli at $10 \mathrm{~Hz}$ with an intertrain interval of $15 \mathrm{~min}$; (5) trains of paired-pulse stimuli adapted from Thiels and colleagues (1994); and (6) trains of paired-burst stimuli adapted from Thiels and colleagues (1996). Experiments using protocols 1-3 were conducted both at baseline intensity and at increased stimulus strength, large enough to evoke a population spike (1-2 mV). Experiments involving paired-pulse (PP) and paired-burst stimulation (PB) were conducted at baseline intensity, except that the current was raised during the LFS treatment to produce a distinct spike in the first response as well as paired-pulse inhibition. To elicit LTP, theta burst stimulation (TBS) was given, consisting of five 30 -msec-long bursts with four pulses at $100 \mathrm{~Hz}$ each, separated by $200 \mathrm{msec}$ and repeated four times at $30 \mathrm{sec}$. The stimulation intensity was increased during TBS to elicit a very small $(<0.5 \mathrm{mV})$ population spike.

\section{LTD induction in the anesthetized rat}

Some of the experiments during the second half of the study were conducted in anesthetized animals and involved testing protocols 1 and 5 outlined above. The following two methods were used.

Method 1. The animals were allowed to recover from surgery for $10 \mathrm{~d}$ and, after verifying that adequate evoked responses had been maintained, were then anesthetized with $55 \mathrm{mg} / \mathrm{kg}$ sodium pentobarbital; placed into the recording cage; and kept at $37.5^{\circ} \pm 0.5^{\circ} \mathrm{C}$ using heating pad, heating lamp, and anal thermoprobe. Depth of anesthesia was monitored continuously throughout the experiment by observing the EEG and testing for reaction to toe pinching every $15-30 \mathrm{~min}$. If necessary, a booster of pentobarbital was administered.

Method 2. The animals were tested in the stereotaxic apparatus on the day of surgery immediately after implanting the electrodes, while being maintained under prolonged pentobarbital anesthesia $(55 \mathrm{mg} / \mathrm{kg})$ and controlled body temperature. After this, the head cap was put in place, and the animals were allowed to recover and used for additional experiments starting $10 \mathrm{~d}$ later.

\section{RESULTS \\ Effects of long trains of LFS}

The occurrence of LTD after 900 single pulses at $1 \mathrm{~Hz}$ in naive (unpotentiated) synapses was tested for in a group of 8 freely moving Long-Evans rats implanted bilaterally with stimulating electrodes in the Schaffer collateral/commissural projections. In half of the animals, the response evoked by stimulating the ipsilateral Schaffer collaterals was used as test input and the contralateral response served as control, whereas reversed assignments were made for the remaining animals. As illustrated in Figure 1, $1 \mathrm{~Hz}$ LFS applied for $15 \mathrm{~min}$ was ineffective at causing synaptic modifications. Increasing the frequency to $5 \mathrm{~Hz}$ and the number of pulses to 3000 stimuli $(10 \mathrm{~min})$ also failed to produce synaptic changes. When the 3000 pulses were given at $10 \mathrm{~Hz}(5$ min) to the same animals and pathways, a substantial depression of the test response was observed $(71.1 \pm 11.6 \%$ of baseline at 5 min). This effect was transient, and the responses recovered to pre-LFS levels within $\sim 3 \mathrm{hr}$ in every case. Control field EPSPs also exhibited an initial decline $(89.4 \pm 10.6 \%$ of baseline $)$ in response to $10 \mathrm{~Hz}$ LFS but returned to baseline within a few minutes after resumption of test stimulation. The protocol described above (three separate LFS treatments at 1, 5, and $10 \mathrm{~Hz}$ given in succession over a period of $90 \mathrm{~min}$ ) was repeated a few days later, but with control and test pathways reversed. This yielded results identical to those of the original test. Combined, the two sets of data demonstrate that LFS at $10 \mathrm{~Hz}$ causes a temporary input-specific depression irrespective of whether the ipsilateral or contralateral pathway serves as test input. In the cases in which the ipsilateral pathway was used as the test input, the initial slope of the field EPSP was $75.3 \pm 4.6 \%$ of baseline at $1 \mathrm{hr}(p<0.001, T(7)=4.74$, paired $t$ test $)$ but recovered to $101.0 \pm 3.4 \%$ within $3.3 \mathrm{hr}$ (Fig. $1 B$ ). An example of a representative experiment is shown in Figure $1 A$. When stimulation at 10 $\mathrm{Hz}$ was given to the contralateral pathway, a depression similar in magnitude and time course was obtained; i.e., the EPSP slope was $81.5 \pm 5.5 \%$ of baseline at $1 \mathrm{hr}(p<0.02, T(7)=2.90)$ and at $99.8 \pm 1.8 \%$ at $3 \mathrm{hr}($ Fig. $1 D)$.

To assess whether the response decrement observed after stimulation at $10 \mathrm{~Hz}$ was the result of a priming effect initiated by the preceding stimulation at lower frequencies, the animals were tested a few days later with $10 \mathrm{~Hz}$ LFS alone. Again, both the ipsilateral and contralateral pathways served as test and control responses, respectively, in a counterbalanced manner across all animals. This protocol produced a temporary homosynaptic depression comparable in amount and time course with that observed when $10 \mathrm{~Hz}$ LFS was preceded by stimulation at 1 and 5 Hz. The field EPSP slope of the ipsilateral test response was $71.6 \pm 6.8 \%$ of baseline at $1 \mathrm{hr}$ and recovered to $101.0 \pm 3.3 \%$ at $3.6 \mathrm{hr}$, whereas the control response exhibited transient depression during the first $5 \mathrm{~min}$ after stimulation at $10 \mathrm{~Hz}$ (Fig. $2 A$ ). Similarly, when the contralateral pathway served as test response, 


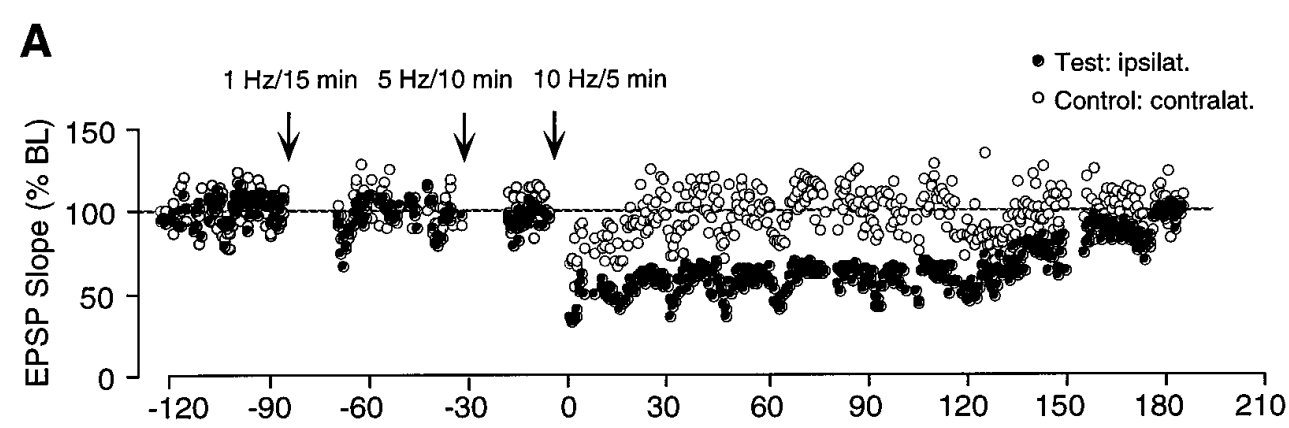

Figure 1. Failure of LFS to induce lasting LTD in area CA1 of the freely moving adult rat. $A$, Individual representative experiment from one of eight animals in which a collection of ipsilateral and contralateral Schaffer collateral/commissural axons was stimulated alternately. Illustrated are changes in slope of the dendritic field EPSP measured in response to single pulse stimulation of the ipsilateral (Test) and contralateral (Control) Schaffer collateral/ commissural pathways before and after delivering LFS to the test input at the times indicated in the graph, i.e., first at $1 \mathrm{~Hz}$, then at $5 \mathrm{~Hz}$, and finally at $10 \mathrm{~Hz}$. $B$, Same as in $A$, illustrated as group data $(n=8)$. Each data point represents the group mean \pm SEM (three averages of four successive responses per pathway and data point) of the initial slope of the dendritic field EPSP expressed as percentage of the baseline. A $5 \mathrm{~min}$ train of LFS at $10 \mathrm{~Hz}$ induced a $2-3 \mathrm{hr}$ lasting depression of test responses without affecting control-evoked heterosynaptic responses to the contralateral stimulating electrode. $C$, Representative population EPSPs in test and control pathway measured in one of the eight animals at different time points before and after the LFS treatments (dashed line). Each waveform represents the average of four successive responses to single-pulse stimulation at $0.05 \mathrm{~Hz}$. The superimposed black line indicates the size of the baseline EPSP recorded before LTD attempts (calibration: 3 $\mathrm{mV} / 10 \mathrm{msec}$ ). $D$, Same as in B, except that the contralateral response $(n=8)$ was used as test pathway and the ipsilateral pathway served as control.
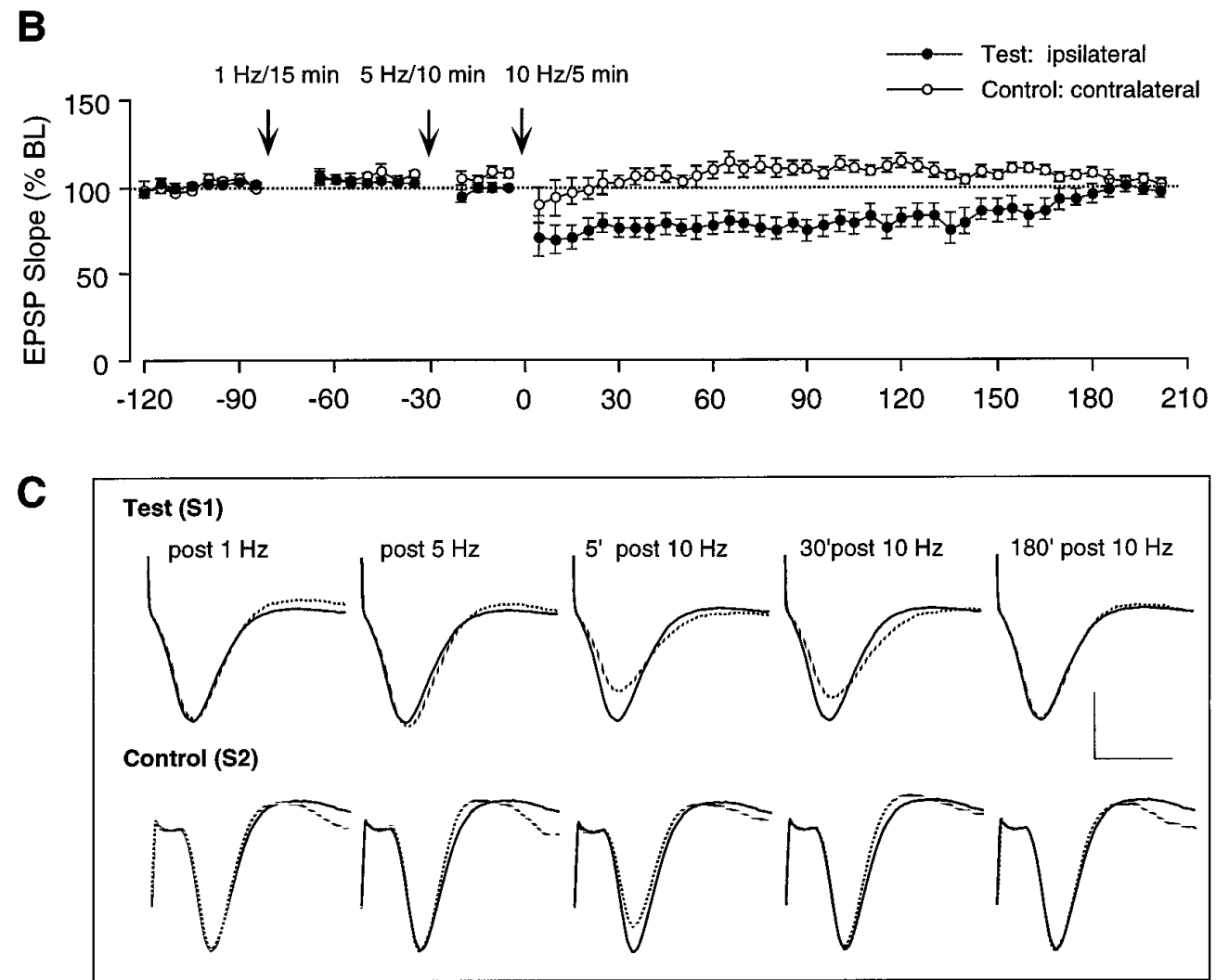

D

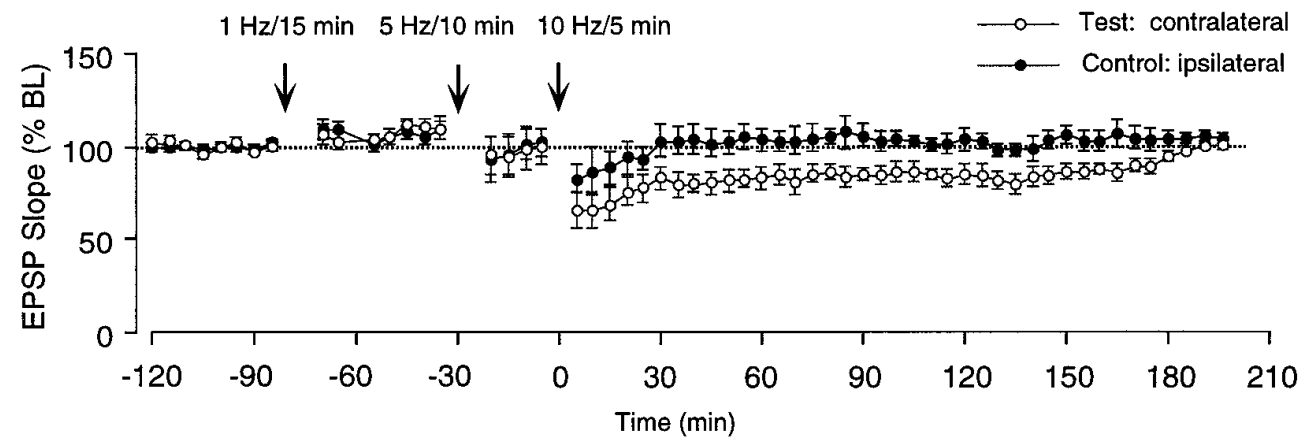

the depression was $77.5 \pm 5.9 \%$ of baseline at $1 \mathrm{hr}$ and back at $100.0 \pm 3 \%$ of baseline at $3.4 \mathrm{hr}$ (data not shown).

To verify that the synapses of these animals were capable of exhibiting long-lasting changes in synaptic efficacy at all, five rats that maintained stable bilateral responses were given TBS $(4 \times 5$ bursts, as described above) to induce LTP. This pattern has been shown previously to produce stable LTP in area CA1 of freely moving rats (Staubli and Lynch, 1987, 1990). As illustrated in Figure $2 B$, TBS induced LTP that persisted for the duration of the experiment $(>24 \mathrm{hr})$. Test EPSPs were $124.8 \pm 10.8 \%$ of baseline at $24 \mathrm{hr}(p<0.05, T(4)=2.38$, paired $t$ test $)$, whereas responses in the control input were $98.4 \pm 3.7 \%$ of baseline.

To test additional LFS protocols, a new group ( $n=6$ animals) was prepared for chronic recording. Of these, three rats with 
A

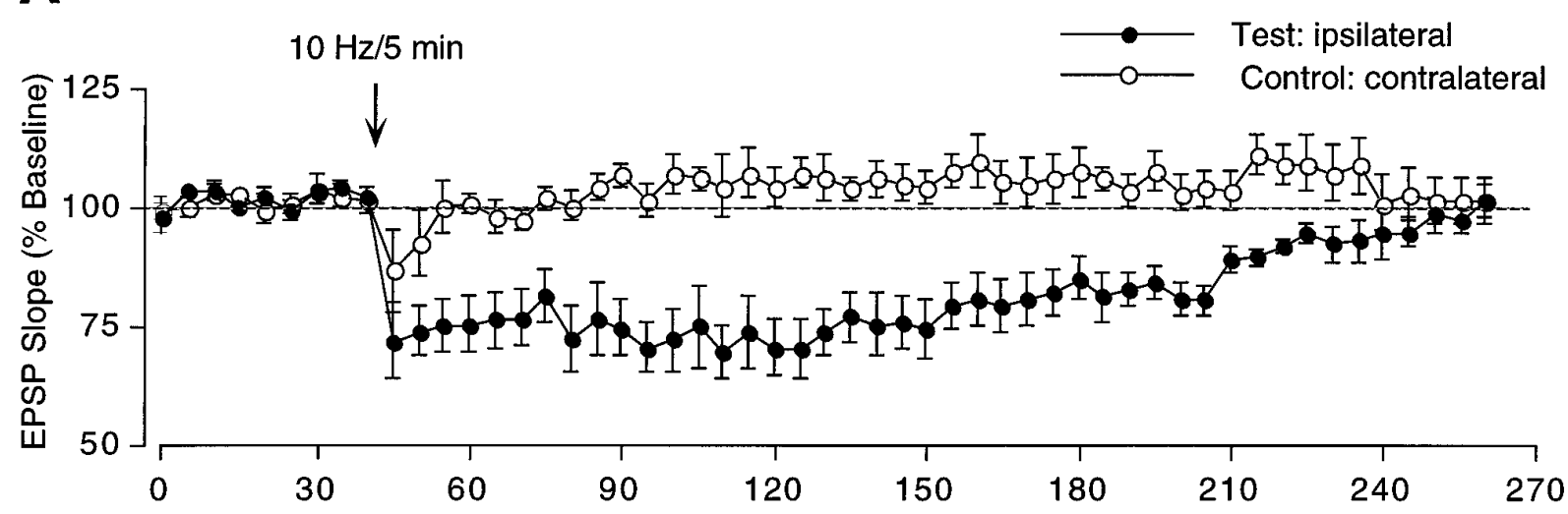

B

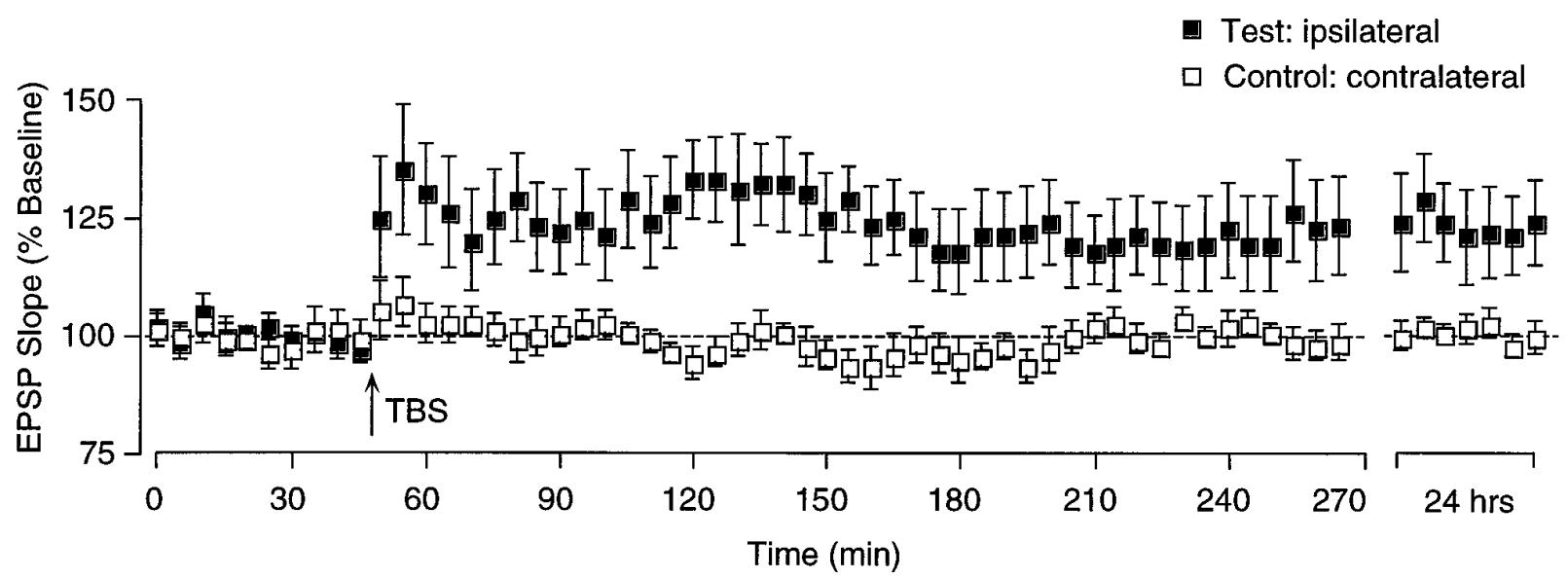

Figure 2. LFS at $10 \mathrm{~Hz}$ produces temporary homosynaptic depression in area CA1 of freely moving adult rats, whereas TBS is capable of eliciting stable LTP in the same synapses. $A$, Group data from eight animals in which temporary LTD was induced by LFS at $10 \mathrm{~Hz}$ ( $5 \mathrm{~min}$ duration) delivered to one of two independent Schaffer collateral/commissural pathways. Each data point represents the group mean \pm SEM (three averages of four successive responses per pathway and data point) of the initial slope of the dendritic field EPSP expressed as percentage of the baseline. $B$, TBS delivered to five of the eight rats shown in $A$, using the same pathway again as test input, induced stable LTP in all animals.

stable bilateral responses were tested with three consecutive 10 $\mathrm{Hz}$ episodes separated by $15 \mathrm{~min}$. As shown in Figure $3 A$, this protocol caused a depression to $56.0 \pm 1.5 \%$ of baseline at $1 \mathrm{hr}$ $(p<0.01, T(2)=9.31)$ and $68.7 \pm 4.3 \%$ at $2 \mathrm{hr}(p<0.01, T(2)$ $=5.44$, paired $t$ test), but subsequently, there was rapid recovery in every case, and $3 \mathrm{hr}$ after the last $10 \mathrm{~Hz}$ episode, the EPSP slope was not significantly different from baseline levels. Three different animals were then tested with two successive high-intensity LFS episodes, the first at $1 \mathrm{~Hz}$ followed $30 \mathrm{~min}$ later by the second at $5 \mathrm{~Hz}$. LFS at $1 \mathrm{~Hz}$ still failed to cause any synaptic modifications, despite the increased stimulus strength (Fig. 3B). In contrast, high-intensity stimulation at $5 \mathrm{~Hz}$ produced a very pronounced depression $(70.0 \pm 8.5 \%$ of baseline slope at $1 \mathrm{hr})$ that recovered within $2 \mathrm{hr}$ but also caused substantial heterosynaptic depression lasting almost $1 \mathrm{hr}$ (Fig. 3B). A few days later, two rats of this group were given high-intensity LFS at $10 \mathrm{~Hz}$, a protocol that resulted in complete erasure of the test response without affecting the control response except for a temporary small decrement (data not shown). In one of these rats, the test response was still visible (20\% of original size) after LFS offset but then disappeared within the next $3 \mathrm{~min}$; in the other animal, the depression was complete at LFS offset. In both animals, there was no sign of recovery from depression, even in recording sessions conducted $5 \mathrm{~d}$ later, and therefore this protocol was abandoned.

\section{Results obtained with patterned stimulation}

Our difficulty in producing activity-dependent LTD reliably prompted us to examine another protocol introduced by Thiels and colleagues (1994), who used a train of paired pulses designed to cause coincident presynaptic excitation and attenuated postsynaptic activation and found that this pattern produced LTD of commissural projections reliably in area CA1 of adult anesthetized rats. Doyere and colleagues (1996) confirmed and extended these findings by showing that the same paired-pulse protocol was capable of inducing LTD in area CA1 of awake animals, provided the initial pulse of the pair was sufficiently strong to cause pairedpulse depression of the evoked response. Therefore, we tested this protocol in a group of 15 rats. After establishing baseline stability across $3 \mathrm{~d}$, paired-pulse stimulation (PP) consisting of 200 pairs of stimuli at $0.5 \mathrm{~Hz}$ was administered, at an increased current intensity that was high enough to evoke an obvious population spike in the first response. The interpulse interval was set between 15 and $25 \mathrm{msec}$, depending on which configuration produced the most prominent paired-pulse inhibition. The ipsilateral pathway served 


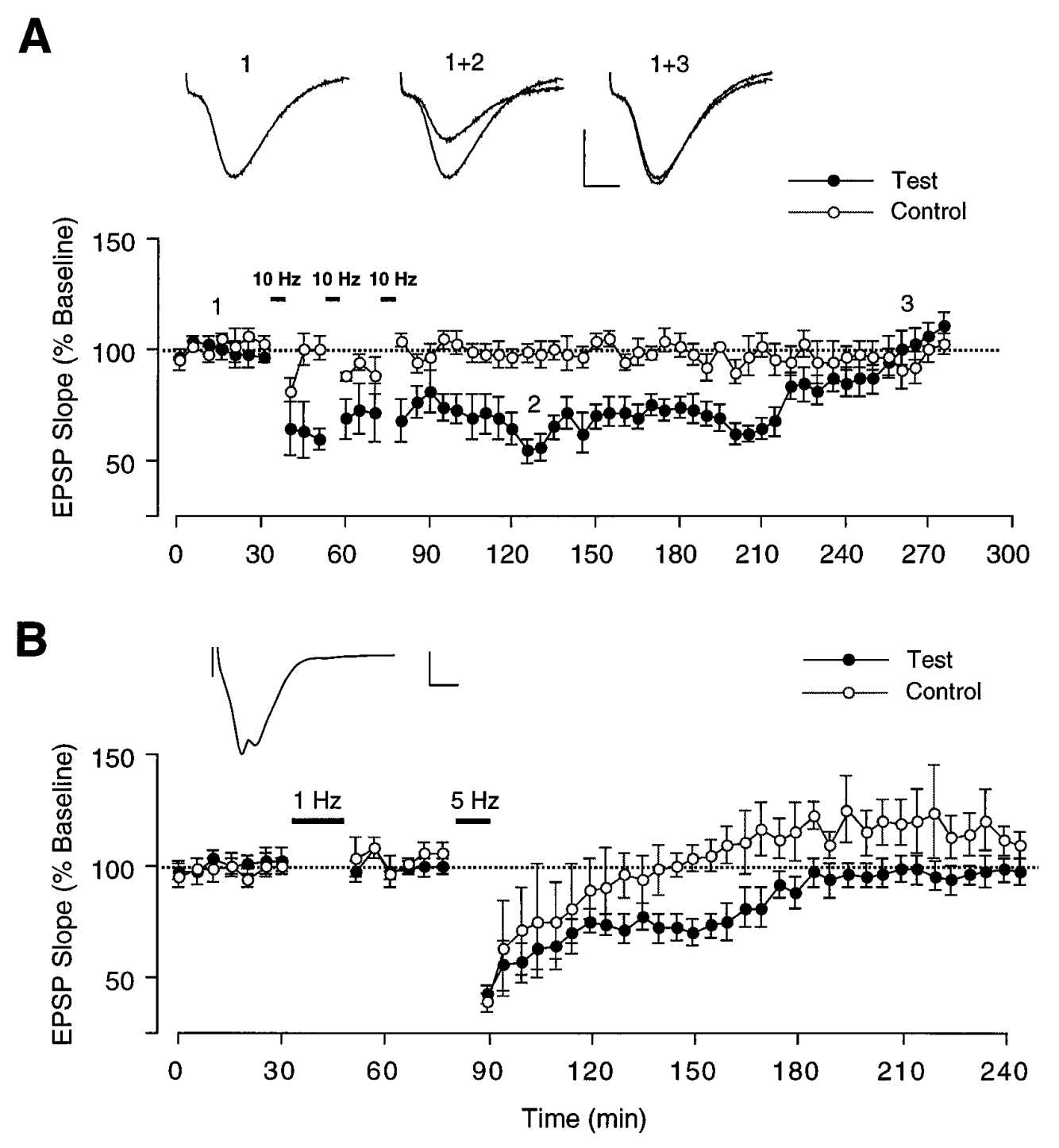

Figure 3. Changes in synaptic transmission induced by repetitive $10 \mathrm{~Hz}$ LFS episodes at low intensity or by a single high-intensity LFS episode at 5 or 10 Hz. $A$, Group data from three rats in which attempts to induce LTD involved delivering three successive $10 \mathrm{~Hz}$ LFS episodes (5 min duration) at $15 \mathrm{~min}$ intervals to the ipsilateral test pathway, whereas the response to the contralateral stimulating electrode served as control. Each data point represents the group mean \pm SEM (three averages of four successive responses per pathway and data point) of the initial slope of the dendritic field EPSP expressed as percentage of the baseline. Inserts show representative individual and superimposed test responses taken at the times indicated in the graph (calibration: 4 $\mathrm{mV} / 5 \mathrm{msec}$ ). $B$, Same as in $A$, except that the stimulus intensity of the test response was increased to produce a population spike, as shown in the insert above (calibration: $4 \mathrm{mV} / 5 \mathrm{msec}$ ). Attempts to induce LTD involved highintensity LFS delivered to the test pathway $(n=3)$ at $1 \mathrm{~Hz}$ for $15 \mathrm{~min}$ and 30 min later at $5 \mathrm{~Hz}$ for $10 \mathrm{~min}$. Each waveform in $A$ and $B$ represents the average of four successive responses to single-pulse stimulation at $0.05 \mathrm{~Hz}$

as test input in all but one of the 15 rats. This paired-pulse protocol caused homosynaptic LTD in three animals that lasted $48 \mathrm{hr}$. The percentage change in test slope was $-22.0+7.2 \%$ at $24 \mathrm{hr}(p<0.5, T(2)=2.76$, paired $t$ test, compared with pre-LFS baseline) and $-15.7 \pm 9.1 \%$ at $48 \mathrm{hr}$ (NS). Control responses were not obviously affected by the paired-pulse treatment in two of the three rats with LTD (the third animal had no measurable control response to start with). On the third day, the depressed test response recovered back to baseline in one rat (the subject without commissural response). In the other two rats, both test and control responses underwent precipitous declines on the third day, probably because of changes in the recording electrode and/or the connections with the head stage (Fig. 4B).

The observation that 12 of the group of 15 rats did not show LTD to the paired-pulse treatment described above suggested that perhaps the mechanisms necessary for LTD induction had not been triggered adequately. Therefore, we retested the 12 rats a few days later using modified stimulation parameters designed to enhance the excitation without compromising paired-pulse inhibition, according to a protocol described by Thiels and colleagues in their follow-up paper (1996). They found that a pattern of "paired-bursts" was capable of inducing robust depression of perforant path-evoked granule cell responses in both anesthetized and nonanesthetized rabbits. We adopted this protocol for area CA1 and delivered 200 pairs of two-pulse bursts at one pair per second, with an interval of 2.5-5 msec between pulses and 10-15 msec between bursts. PBs produced homosynaptic LTD in 5 of the 12 rats tested. In two of these, the depression lasted $<24 \mathrm{hr}$, but in three rats, it was still present $72 \mathrm{hr}$ later (Fig. $4 C$ ). The percentage change in test slope was $-22 \pm 2.8 \%$ at $3 \mathrm{hr}(p<$ $0.001, T(4)=7.93$, paired $t$ test, compared with pre-LFS baseline), and $-21.7 \pm 6.9 \%$ at $72 \mathrm{hr}(p<0.05, T(2)=3.11$, paired $t$ test, compared with pre-LFS baseline). Control pathways were initially also affected by PB stimulation but then recovered back to baseline levels within $1 \mathrm{hr}$.

To summarize, 8 of 15 rats exhibited depression in response to stimulation with paired pulses or PBs. The decrement in synaptic transmission lasted for at least $3 \mathrm{hr}$ in all eight of these animals, 48 hr in six, and $72 \mathrm{hr}$ in three (Fig. 4A).

\section{Interactions with ampakines and barbiturates}

We also examined whether LTD induction might be enhanced in animals treated with allosteric AMPA receptor facilitating drug 1-(quinoxolin-6-ylcarbonyl)piperidine (BDP-12, Cortex Pharmaceuticals). BDP-12, which belongs to a family of compounds called ampakines, which produce changes in AMPA receptor kinetics 
A

- Test (S1)
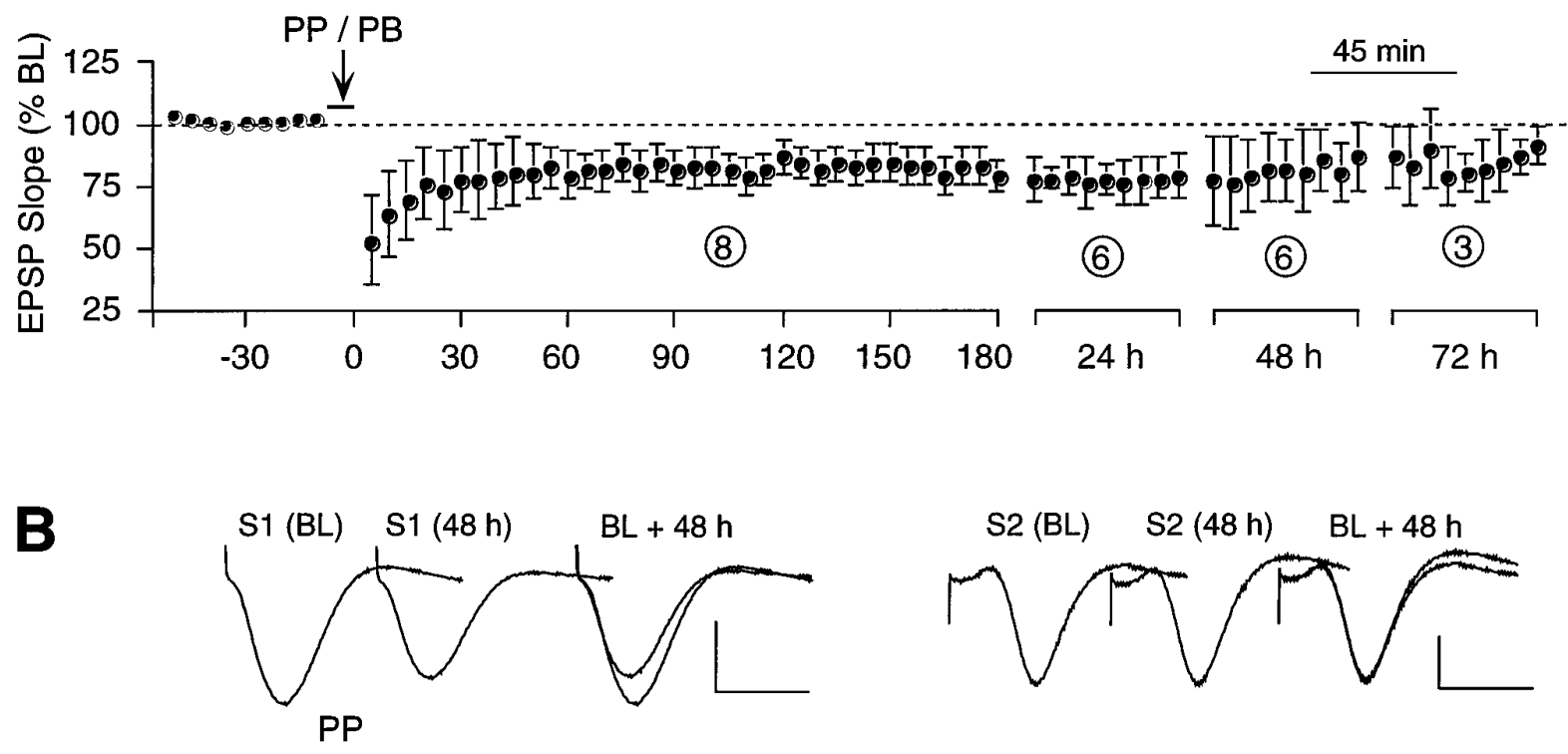

$B L+48 h$
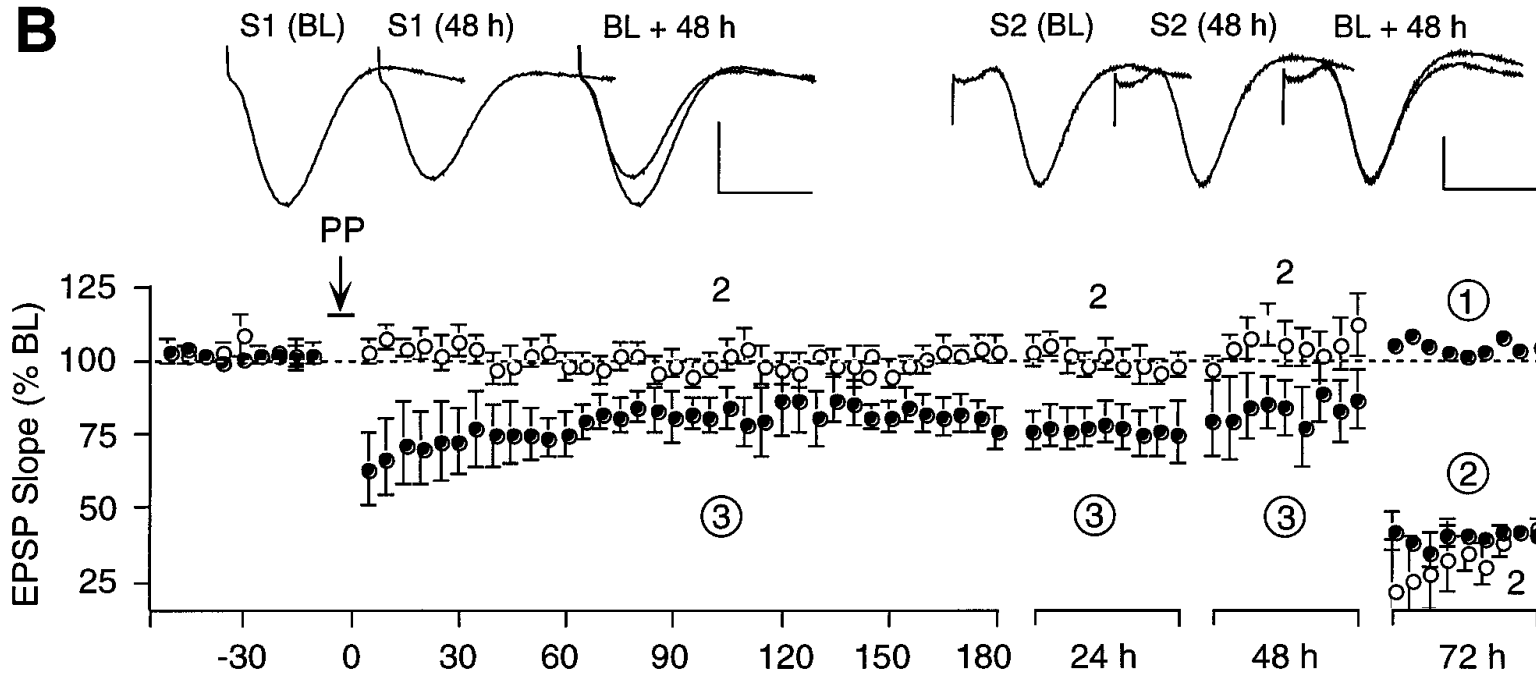

2

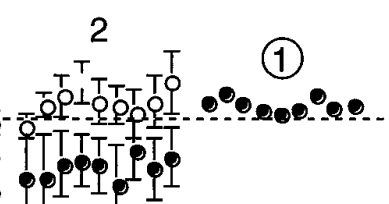

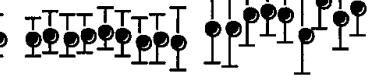

(3)

(2)

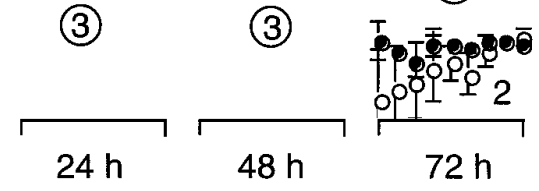

C

- Test (S1)

- Control (S2)

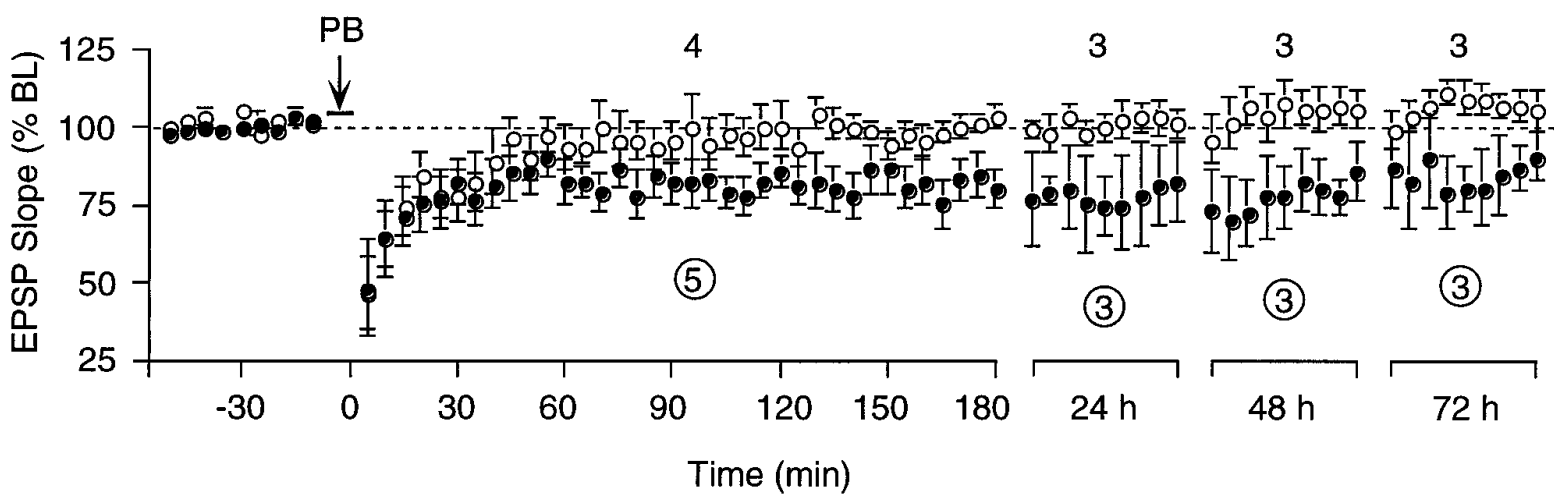

Figure 4. Ability of paired-pulse $(P P)$ and paired-burst stimulation $(P B)$ to induce LTD in area CA1 of freely moving adult rats. $A$, Group data summarizing the 8 of 15 animals in which either PP or PB stimulation caused synaptic depression. Values in a circle correspond to the number of test pathways used to calculate the group mean $( \pm$ SEM) and represent the initial slope of the dendritic field EPSP expressed as percentage of the baseline. Each data point consists of three averages of four successive responses per pathway. The percentage change in test slope compared with pre-LFS baseline was $-23.5 \pm 2.0 \%$ at $3 \mathrm{hr}[p<0.001, T(7)=11.6],-26.8 \pm 7.3 \%$ at $24 \mathrm{hr}[p<0.01, T(5)=3.68],-19.3 \pm 5.6 \%$ at $48 \mathrm{hr}[p<0.01, T(5)=3.51]$, and $-21.7 \pm 7.0$ at $72 \mathrm{hr}[p<0.05, T(2)=3.11] . B$, Summary graph of the subset of three animals from the group of eight depicted in $A$ that showed depression in response to PP stimulation. Values in a circle (Test) and without circle (Control) correspond to the number of pathways available within each time period to calculate the group mean $( \pm \mathrm{SEM})$. Inserts show representative individual and superimposed test $(S 1)$ and control responses $(S 2)$ taken at the times indicated (calibration: $3 \mathrm{mV} / 5 \mathrm{msec}$ for $\mathrm{S} 1,2 \mathrm{mV} / 5 \mathrm{msec}$ for S2). Each waveform represents the average of four successive responses to single-pulse stimulation at $0.05 \mathrm{~Hz}$. $C$, Summary graph of the five of eight animals that exhibited LTD in response to PB but had not shown depression in response to PP stimulation earlier. Values in a circle (Test) and without circle (Control) correspond to the number of pathways available within each time period to calculate the group mean $( \pm$ SEM). 

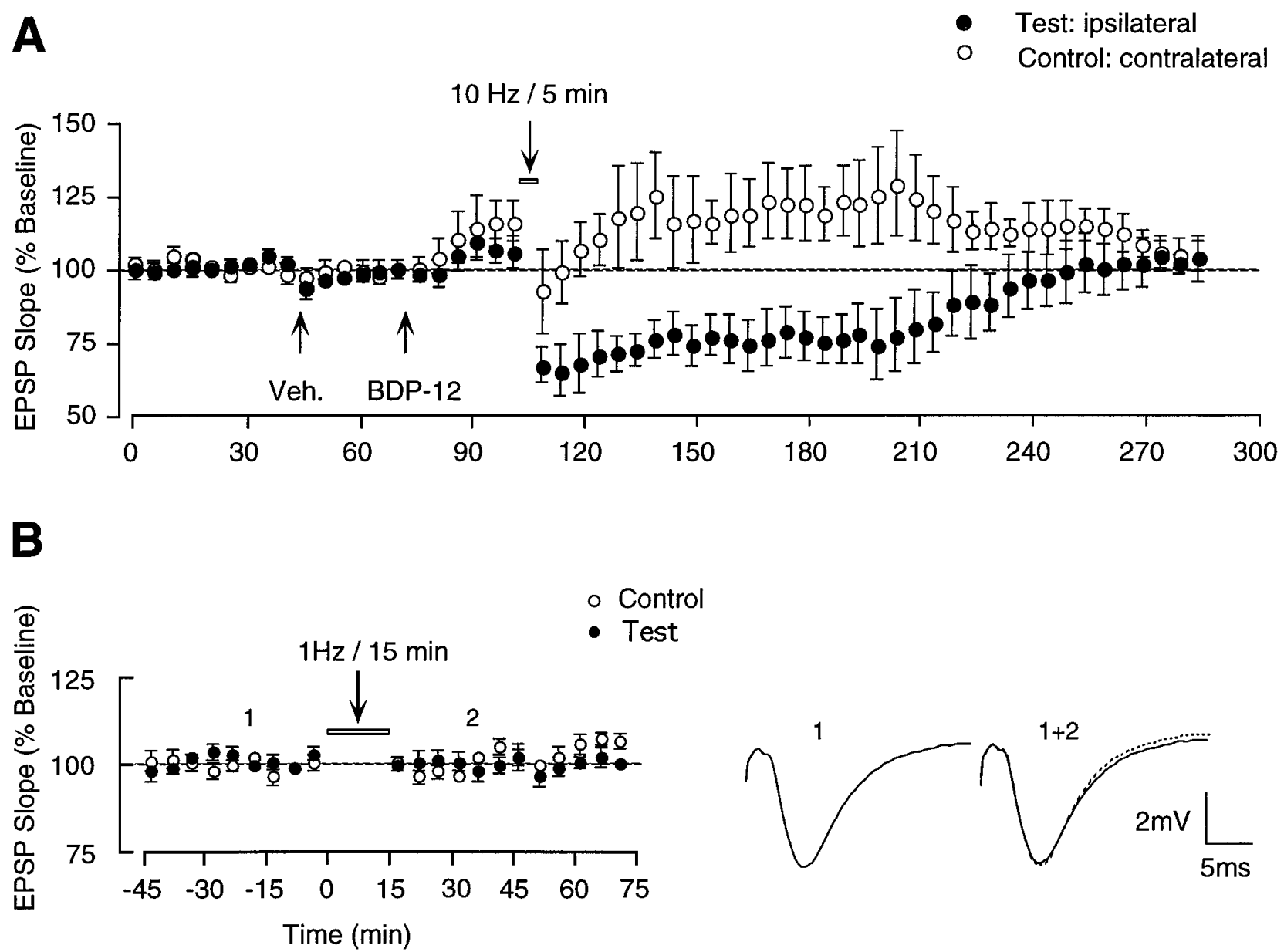

Figure 5. Effect of LFS on synaptic transmission during barbiturate anesthesia and in animals treated with AMPA receptor-facilitating drug. $A$, LFS (10 $\mathrm{Hz}$ ) delivered to one of two independent Schaffer collateral/commissural pathways in a group of six animals 30 min after injection of allosteric AMPA receptor modulator BDP-12 did not facilitate amount or duration of the ensuing depression compared with control conditions (compare with Fig. $2 A$ ). Each data point represents the group mean \pm SEM (three averages of four successive responses per pathway and data point) of the initial slope of the dendritic field EPSP expressed as percentage of the baseline. B, left, Nine hundred pulses at $1 \mathrm{~Hz}$ delivered under barbiturate anesthesia to one of two independent Schaffer collateral/commissural pathways failed to produce a depression of the test response in 12 of 15 animals. Each data point represents the group mean \pm SEM (three averages of four successive responses per pathway and data point) of the initial slope of the dendritic field EPSP expressed as percentage of the baseline. B, right, Waveforms represent typical test responses (average of four) taken before (solid line) and after (dotted line) LFS. Note that there is no change in slope or amplitude induced by LFS. Three of this group of 15 animals did show LTD, but the depression was accompanied by persistent heterosynaptic effects (data not shown; see text).

(Arai et al., 1994, 1996a,b) and thereby enhance synaptic responses and LTP induction (Staubli et al., 1994), has been used successfully in studies of LTP reversal in which it significantly facilitated depotentiation induced by stimulation at $5 \mathrm{~Hz}$ (Staubli and Chun, 1996a,b). To test whether BDP-12 might be similarly effective at promoting LTD, six rats with stable responses received intraperitoneal injections, first of saline and then BDP-12 (50 $\mathrm{mg} / \mathrm{kg}$ ) $30 \mathrm{~min}$ before administering LFS at $10 \mathrm{~Hz}$ to the ipsilateral input. That BDP-12 had crossed the blood-brain barrier and was effective at facilitating transmission was evident from the change in size and waveform of evoked responses, an effect that began 5 min after drug injection and lasted for the duration of 2-3 hr (see control pathway in Fig. $5 A$ ). However, despite the presence of the drug, the depression produced by stimulation at $10 \mathrm{~Hz}$ was not long-lasting. Test EPSPs were $73.8 \pm 8.6 \%$ of baseline at $1 \mathrm{hr}(p<0.02, T(5)=2.94$, paired $t$ test $)$ and steadily recovered back to baseline levels over $3 \mathrm{hr}(103.6 \pm 4.8 \%$ at $3 \mathrm{hr})$.

The next series of experiments was conducted in anesthetized animals and is summarized in Figure $5 B$. According to a recent report, LFS at $1 \mathrm{~Hz}$ (900 pulses) was effective at reliably inducing homosynaptic depression when delivered to the ipsilateral Schaffer collateral input in adult rats under barbiturate anesthesia (Heynen et al., 1996). To test this protocol, we used four animals that had been implanted earlier using our standard coordinates (which are slightly different from those described by Heynen; see Materials and Methods) and reanesthetized them with pentobarbital for the experiment. Based on Heynen's report that LFS at 1 $\mathrm{Hz}$ produces little if any LTD of the commissural input, the ipsilateral pathway was chosen as test input in all but one rat that had lost its ipsilateral response. LFS produced a lasting depression $(3 \mathrm{hr})$ of the commissural response in this latter animal, but no short-term or long-term synaptic changes were evident in the other three rats. This same protocol was then tested in 11 additional animals with bilateral stimulating electrodes placed according to the coordinates described by Heynen and colleagues (1996). LFS was delivered on the day of surgery to the ipsilateral test input immediately after completion of electrode implantation, with the animals under stable anesthesia and body temperature 
conditions. Again, the stimulation had no impact in the majority of rats, except in two that responded with a marked depression of the ipsilateral response. However, the control pathway was also affected in both cases, exhibiting lasting heterosynaptic depression in one rat and potentiation in the other rat. In sum, $20 \%(3 / 15)$ of the rats given LFS at $1 \mathrm{~Hz}$ under barbiturate anesthesia showed LTD of significant magnitude; however, the observed depression did not appear to be synapse-specific (Fig. 5B).

In a final step, to determine whether the success rate of inducing LTD to patterned low-frequency trains might improve if the experiment was conducted under barbiturate anesthesia, we tested seven newly implanted rats on the day of surgery in the stereotaxic apparatus by delivering 200 pairs of stimuli (stimulus interval $25 \mathrm{msec}$ ) every $2 \mathrm{sec}$ to the ipsilateral test pathway. The current intensity was increased during paired-pulse treatment to ensure a distinct population spike in the first evoked response as well as strong paired-pulse inhibition within the pair. Despite the fact that the paired-pulse depression of the population spike obtained under anesthesia was very pronounced in every case $(>50 \%)$ compared with our earlier observations in awake animals in which the inhibition ranged between 20 and $40 \%$, each of the seven animals responded with only a temporary $(<15 \mathrm{~min})$ depression that affected both the ipsilateral and contralateral pathways in a comparable manner (data not shown).

\section{DISCUSSION}

We tested six stimulation paradigms that consisted of either uniform trains of single pulses or patterned stimulation trains applied at variable low frequencies and either low- or highintensity current. Low-intensity, prolonged stimulation at $1-5 \mathrm{~Hz}$ did not cause any changes but at $10 \mathrm{~Hz}$ reliably elicited homosynaptic depression, an effect that dissipated within $3 \mathrm{hr}$ in every case (Figs. 1, 2A, 3A). High-intensity, prolonged stimulation at $1 \mathrm{~Hz}$ still had no impact, but at $5 \mathrm{~Hz}$ caused a depression encompassing both pathways and recovering within 1-3 hr (Fig. 3B). Highintensity current at stimulation of $10 \mathrm{~Hz}$ led to a complete and apparently permanent loss of the homosynaptic response, an effect that seemed pathological and of uncertain relevance to normal synaptic operations. Overall, the impact of uniform trains of low-frequency pulses was more pronounced the smaller interpulse intervals and the higher the current intensity. However, in no case did the depression last much longer than $3 \mathrm{hr}$.

Switching to a patterned stimulation protocol increased the likelihood of inducing LTD. Specifically, trains patterned as a series of paired-pulses produced LTD that persisted for $48 \mathrm{hr}$, with minimal effects on heterosynaptic responses (Fig. 4B), but only in a small percentage of cases, i.e., 20\% (3/15). The probability of producing more persistent LTD improved when trains patterned as a series of PBs were used; almost half of the subjects $(5 / 12)$ who until then had not responded to any stimulation protocol now showed LTD, with the depression lasting $>72 \mathrm{hr}$ in three cases. However, in contrast to paired-pulse trains, PB trains had the additional effect of causing heterosynaptic depression, which in most rats was very substantial at the outset and then recovered slowly over the next two hr (Fig. 4C). Persistent heterosynaptic depression as an obligatory companion in cases in which the homosynaptic decrement is pronounced and longlasting would profoundly affect hypotheses regarding LTD as information storage mechanism.

The chronic recording experiments described above used a second stimulating electrode activating nonoverlapping afferents terminating on the same population of dendrites as those stimu- lated by the test electrode to control for input specificity of LTD. Although Thiels and colleagues were able to induce synaptic depression both in area CA1 of the anesthetized rat and in dentate gyrus of the awake rabbit, they did not establish that the depression was homosynaptic or confirm that it lasts $>3 \mathrm{hr}$. Similarly, the reports by Doyere and colleagues (1996) did not describe a control pathway. In contrast, Heynen and colleagues (1996) did include a control input in their study on LTD in anesthetized rats and also recorded for several hours in some subjects. However, as outlined above, standard LFS as used by Heynen was relatively ineffective in causing LTD in the freely moving animals in the present study.

Consistent with previous results, the present findings suggest that the optimal parameters for inducing LTD differ between in vitro and in vivo CA1 preparations, i.e., the standard protocol of 900 pulses at $1 \mathrm{~Hz}$ introduced by Dudek and Bear (1992), which is well suited to produce activity-dependent depression in slices from immature and adult rats (Dudek and Bear, 1993; Staubli and $\mathrm{Xi}, 1996)$, failed to elicit LTD in the awake and anesthetized rat in our hands, a result in agreement with observations by others (Errington et al., 1995; Doyle et al., 1997; but see also Heynen et al., 1996). The two studies with negative results cited above used Sprague Dawley animals, as did the one by Heynen, whereas the present experiments were performed in Long-Evans rats. Thus, it is unlikely that strain differences account for the inconsistent results across studies. With regard to relative effects in vitro versus in vivo, it will be of interest to test whether the low-frequency patterns found in the present study to induce synaptic depression in vivo also elicit LTD in the slice preparation. Relevant to this, it should be noted that TBS, a stimulation protocol well suited for LTP induction in vitro (Larson et al., 1986; Larson and Lynch, 1986), induces stable potentiation in area CA1 of the freely moving rat (Staubli and Lynch, 1987, 1990).

LTD has a number of features in common with LTP reversal, or depotentiation, and the two phenomena are sometimes equated in the recent literature. Previous studies on LTP reversal suggest that the effects are quite distinct (Staubli and Lynch, 1990; Staubli and Chun, 1996a,b). Reversal is achieved readily with a short episode of low-intensity, naturalistic stimulation $(5 \mathrm{~Hz}$ or theta pulse stimulation) both in vitro and in the freely moving animal, is not dependent on NMDA receptors, and can be obtained only for a limited time after LTP induction; moreover, naive synapses remain unchanged by theta pulse stimulation. LTD, as shown by a number of investigators, requires prolonged trains of lowfrequency pulses at high intensity (at least in the adult brain) and is obtained irrespective of whether the synapses are naive or potentiated; it also involves NMDA receptors. The present results extend these differences by showing that a pharmacological manipulation ("up modulation" of AMPA receptors) that enhances depotentiation (Staubli and Chun, 1996a,b) has no effect on LTD, whereas a second treatment (barbiturate anesthesia), which does not affect reversal (Barrionuevo et al., 1980), reduces the likelihood of obtaining depression. Thus, it appears that LTD of potentiated synapses by prolonged LFS and depotentiation triggered by short episodes of theta pulse stimulation are manifestations of different cellular processes. It remains to be determined which of these two laboratory effects, if either, occurs during behavior and is used by the brain to acquire and store information. In any case, any correspondences in learning and memory for the two phenomena are likely to be very different. 


\section{REFERENCES}

Arai A, Kessler M, Xiao P, Ambros-Ingerson J, Rogers G, Lynch G (1994) A centrally active drug that modulates AMPA receptor gated currents. Brain Res 638:343-346.

Arai A, Kessler M, Ambros-Ingerson J, Quan A, Yigiter E, Rogers G, Lynch G (1996a) Effects of a centrally active benzoylpyrrolidine drug on AMPA receptor kinetics. Neuroscience 75:573-585.

Arai A, Kessler M, Rogers G, Lynch G (1996b) Effects of a memory enhancing drug on AMPA receptor currents and synaptic transmission in hippocampus. J Pharmacol Exp Ther 278:627-638.

Barrionuevo G, Schottler F, Lynch G (1980) The effects of repetitive low frequency stimulation on control and "potentiated" responses in the hippocampus. Life Sci 27:2385-2391.

Bear MF, Malenka RC (1994) Synaptic plasticity: LTP and LTD. Curr Opin Neurobiol 4:389-399.

Bienenstock EL, Cooper LN, Munro PW (1982) Theory for the development of neuron selectivity: orientation specificity and binocular interaction in visual cortex. J Neurosci 2:32-48.

Doyere V, Errington ML, Laroche S, Bliss TVP (1996) Low-frequency trains of paired stimuli induce long-term depression in area CA1 but not in dentate gyrus of the intact rat. Hippocampus 6:52-57.

Doyle CA, Cullen WK, Rowan MJ, Anwyl R (1997) Low-frequency stimulation produces homosynaptic depotentiation but not long-term depression of synaptic transmission in the adult rat hippocampus in vivo. Neuroscience 77:75-85.

Dudek SM, Bear MF (1992) Homosynaptic long-term depression in area CA1 of hippocampus and the effects of NMDA receptor blockade. Proc Natl Acad Sci USA 89:4363-4367.

Dudek SM, Bear MF (1993) Bidirectional long-term modification of synaptic effectiveness in the adult and immature hippocampus. J Neurosci 13:2910-2918.

Errington ML, Bliss TVP, Richter-Levin G, Yenk K, Doyere V, Laroche S (1995) Stimulation at $1-5 \mathrm{~Hz}$ does not produce long-term depression or depotentiation in the hippocampus of the adult rat in vivo. J Neurophysiol 74:1793-1799.
Heynen AJ, Abraham WC, Bear MF (1996) Bidirectional modification of CA1 synapses in the adult hippocampus in vivo. Nature 381:163-166.

Hopfield (1982) Neural networks and physical systems with emergent collective computational abilities. Proc Natl Acad Sci USA 79:2554-2558.

Larson J, Lynch G (1986) Induction of synaptic potentiation in hippocampus by patterned stimulation involves two events. Science 232:985-988.

Larson J, Wong D, Lynch G (1986) Patterned stimulation at the theta frequency is optimal for the induction of hippocampal long-term potentiation. Brain Res 386:347-350.

Staubli U, Chun D (1996a) Factors regulating the reversibility of longterm potentiation. $\mathrm{J}$ Neurosci 16:853-860.

Staubli U, Chun D (1996b) Proactive and retrograde effects on LTP produced by theta pulse stimulation: mechanisms and characteristics of LTP reversal in vitro. Learn Memory 3:96-105.

Staubli U, Ji Z-X (1996) The induction of homo- vs. heterosynaptic LTD in area CA1 of hippocampal slices from adult rats. Brain Res 714:169-176.

Staubli U, Lynch G (1987) Stable hippocampal long-term potentiation elicited by "theta" pattern stimulation. Brain Res 435:227-234.

Staubli U, Lynch G (1990) Stable depression of potentiated synaptic responses in the hippocampus with $1-5 \mathrm{~Hz}$ stimulation. Brain Res 513:113-118.

Staubli U, Perez Y, Xu F, Rogers G, Ingvar M, Stone-Elander S, Lynch G (1994) Centrally active modulators of glutamate receptors facilitate the induction of LTP in vivo. Proc Natl Acad Sci USA 91:11158-11162.

Staubli U, Chun D, Xu F, Ji Z-X (1995) Reversal of long-term potentiation is different from long-term depression. Soc Neurosci Abstr 21:1098.

Thiels E, Barrionuevo G, Berger T (1994) Excitatory stimulation during postsynaptic inhibition induces long-term depression in hippocampus in vivo. J Neurophysiol 72:3009-3016.

Thiels E, Xie X, Yeckel MF, Barrionuevo G, Berger TW (1996) NMDA receptor-dependent LTD in different subfields of hippocampus in vivo and in vitro. Hippocampus 6:43-51. 